\title{
Introduction to special issue on Software Engineering Aspects of Green Computing (SEAGC)
}

It is our pleasure to serve as guest editors of this special issue on Software Engineering Aspects of Green Computing (SEAGC).

Since the early days of ICT systems, a significant amount of research has been performed in developing efficient algorithms from the perspectives of required memory space and execution time. Due to the growing usage of the ICT in every aspects of our life, such as the increased adoption of mobile devices, energy-efficient computing has been extensively studied during the last decade. Modern hardware requires more materials for production, consumes energy, and requires resources for disposal. Nevertheless, by increasing the intelligence of software in the production and operational phases, software can play a major role in achieving greener computing environments.

Software is frequently blamed as the cause of the energy increase in the production of hardware since software has been the driver for new and faster hardware. Nevertheless, if designed properly, software can avoid early disposal of hardware as well. The "right" choice of algorithms, software development processes, development environments such as architecture description languages, testing environments, they all can have a strong impact in reducing resource consumption.

From the perspective of software engineering, various research topics can be defined in the area of green computing. This includes, but not limited to, (a) defining green software development processes, which require less resources to develop software; (b) defining green software development tools; (c) developing green algorithms; and (d) adopting software systems in different application domains for the purpose of controlling hardware so that energy-efficient computing can be achieved.

These areas of research categories in green computing can be further divided according to the sub-areas of software engineering, such as requirements for energy-efficient systems, model-driven development of green software, programming languages for energy-efficient computing, energy-efficient database techniques, etc. In this special issue, we intended to emphasize the need for studying green computing from the perspective of software engineering methods and tools.

Aligning with the high-quality standards of SUSCOM, in this special issue we have applied a rigorous review process. The papers were reviewed by three to four experts and discussed extensively. Among the submitted papers, two papers entitled
"A systematic literature review on Energy Efficiency in Cloud Software Architectures", and "A Study of Energy-Aware Implementation Techniques: Redistribution of Computational Jobs in Mobile Apps" were accepted to be included in this special issue.

In the first paper, the authors perform a systematic literature review by investigating cloud software architectures with energyefficiency as the primary concern. The review forms a strong base for further systematic research in this area The second paper uses measurements to investigate the impact of the implementation techniques Method Reallocation and Method Offloading in Java and $C$ in different execution scopes of the Android OS runtime and in remote servers. As a result, the authors identified the cases in which it is convenient to reallocate the processing job, and when it is advisable to offload it to an external infrastructure.

We believe that the selected papers lay a strong ground for the research and as such will inspire other researchers in the area of software engineering aspects of green computing. We hope that the readers will find the papers in this special issue informative and useful.

The guest editors wish to thank the authors of all the submitted manuscripts, without them this special issue would not be possible. They also thank the reviewers who provided a thorough evaluation of the submitted manuscripts in a timely manner. We appreciate the assistance of the Special Issues Editor-in-Chief, Behrooz Shirazi, and the SUSCOM editorial staff throughout the process of bringing out the special issue.

Somayeh Malakuti* Technical University of Dresden, Germany

Wolfgang Lohmann Independent Researcher, St. Gallen, Switzerland

Mehmet Aksit University of Twente, The Netherlands

* Corresponding author. E-mail addresses: somayeh.malakuti@tu-dresden.de (S. Malakuti), wolfgang.lohmann@gmail.com (W. Lohmann), m.aksit@utwente.nl (M. Aksit). 